\title{
PARASITISMO DE Meloidogyne enterolobii EM ESPÉCIES DE MYRTACEAE ${ }^{1}$
}

\author{
LUIZA SUELY SEMEN MARTINS ${ }^{2}$, ROSIMAR DOS SANTOS MUSSER ${ }^{3}$, \\ ALINE DAS GRAÇAS SOUZA ${ }^{4}$, LUCIANE VILELA RESENDE ${ }^{5}$, WILSON ROBERTO MALUF ${ }^{6}$
}

RESUMO - A meloidoginose da goiabeira, causada por $M$. enterolobii (= M. mayaguensis), é hoje considerado o principal problema fitossanitário desta cultura em todo o País, pois sua incidência resulta em acentuada queda de produtividade e, na maioria das vezes, a morte das plantas em médio prazo. Visando a seu controle, objetivou-se neste trabalho identificar fontes de resistência em goiabeira Psidium guajava L e araçazeiro Psidium sp.. Foram avaliados onze genótipos oriundos do Câmpus da Universidade Federal de Lavras (UFLA) e de regiões próximas ao município de Lavras (MG). As sementes foram coletadas de frutos fisiologicamente maduros e germinadas em piscinas hidropônicas. Ao atingirem $12 \mathrm{~cm}$ de altura, foram transferidas para casa de vegetação, em vasos individuais, e mantidas a temperatura entre $18-38^{\circ} \mathrm{C}$. Após quinze dias, foram inoculados individualmente com suspensão de 10.000 ovos e juvenis de segundo estádio (J2) de M. enterolobii e avaliadas após 120 dias. As reações dos hospedeiros foram enquadradas nos parâmetros estabelecidos pelo fator de reprodução FR, estimado pelo quociente $\mathrm{Pf} / \mathrm{Pi}$, em que Pf representa a população final e Pi a população inicial. O delineamento utilizado foi o inteiramente casualizado, com 11 genótipos, uma espécie de nematoide e seis repetições, sendo duas plantas em cada genótipo adicionadas como controle. Quatro meses depois, foram avaliados os teores foliares de macro e micronutrientes e o grau de infecção de cada genótipo. A produção média de ovos e J2 de M. enterolobii variou de 200 a 428.146,1, e os fatores de reprodução (FR) médios variaram de 0,02(ALU1) a 42,81(A-PASTO). Os genótipos de araçazeiro (Psidium spp.) AUFLA1, AUFLA4, AUFLA5 e APASTO e os genótipos de goiabeira (P. guajava) G-ROXA e G-AMAR foram considerados suscetíveis. Os genótipos de araçazeiro ALU1, ALU2, ALU3, AROXO-C e AROXO-U mostraram-se resistentes, reforçando a necessidade de trabalhos complementares com essas espécies para uso como porta-enxerto em cultivares comerciais de goiabeira. A absorção de macro e micronutrientes é alterada quando os genótipos de Psidium spp. são inoculados com o fitonematoide $M$. enterolobii.

Termos para indexação: Goiaba, araçá, nematoide, macronutrientes, micronutrientes.

\section{PARASITISM OF Meloidogyne enterolobii IN MYRTACEAE SPECIES}

\begin{abstract}
Guava tree root-knot, caused by $M$. enterolobii (= M. mayaguensis), is today regarded as the main phytosanitary problem of this crop all over the country, for its incidence results into marked yield fall and most of the time, the death of the plant on a medium term. Aiming at its control, it was intended in this study to identify resistance sources on guava tree, Psidium guajava L and Surinam cherry tree, Psidium sp. Eleven genotypes coming from the campus of the Federal University of Lavras (UFLA - Universidade Federal de Lavras) and regions close to Lavras (MG) were evaluated. The seeds were collected from physiologically ripe fruits and germinated in hydroponic pools. Reaching $12 \mathrm{~cm}$ high, they were transferred to the greenhouse in individual pots and kept at the temperature between 18 to $38^{\circ} \mathrm{C}$. After fifteen days, they were inoculated with a suspension of 10,000 eggs and J2 of $M$. enterolobii and evaluated after 120 days. The hosts' reactions were fitted in the parameters established by the reproduction factor FR, estimated by the Pf/Pi quotient, where Pf stands for the final population and Pi the initial population and by the gall index (IG) and index of egg mass (IMO) through the score scale of the International Meloidogyne Project (IMP). The design utilized was the completely randomized with 11 genotypes, one nematode species and six replicates, that is, two plants in each genotype being added as a control. Four months later, the leaf contents of macro and micronutrients and degree of infection of each genotype were evaluated. The average yield of eggs and J2 of $M$. enterolobii ranged from 200 to 428,146.1 and the average reproduction factors (FR) varied from 0.02(ALU1) to 42.81(A-PASTO). The genotypes of Surinam cherry (Psidium spp.) AUFLA1, AUFLA4, AUFLA5 and APASTO and the genotypes of guava (P. guajava) G-ROXA and G-AMA were regarded as susceptible. The Surinam cherry genotypes ALU1, ALU2, ALU3, AROXO-C and AROXO-U proved resistant. Reinforcing the need for further studies with these species using as rootstock in commercial guava cultivars. The absorption of macro and micronutrients is altered when the genotypes of Psidium spp. are inoculated with phytonematode $M$. enterolobii.
\end{abstract}

Index terms: Guava, araça, nematodes, macronutrients, micronutrients.

1(Trabalho 172-11). Recebido em: 06-06-2011. Aceito para publicação em: 10-05-2013.

${ }^{2}$ P.h.D. Professora. Associada do depto de biologia -UFRPE. Campus Universitário-Recife-PE. E-mail: luiza@db.ufrpe.br

${ }^{3}$ P.h.D. Professora.Associada do depto de Fitotecnia -UFRPE. Campus Universitário - Recife-PE. E-mail: rosimar.musser@gmail.com

${ }^{4}$ Doutoranda em Fitotecnia -UFLA-Campus Universitário Lavras-MG. Autor para correspondência. E-mail: alinedasgracas@yahoo.com.br ${ }_{5}^{5}$ h.D. Professora Associada do depto de Fitotecnia -UFLA- Campus Universitário - Lavras-MG. E-mail: vilela@dag.ufla.br ${ }^{6}$ Ph.D. Professor Titular do depto de Fitotecnia -UFLA- Campus Universitário - Lavras-MG. E-mail: wrmaluf@ufla.br 


\section{INTRODUÇÃO}

A goiabeira (Psidium guajava L.) apresenta-se como uma frutífera de alta rentabilidade e com grandes possibilidades de expansão nos mercados interno e externo (GOMES, 2007). Em 2001, a produção brasileira de goiaba foi de 281.102 toneladas, apresentando discreto aumento em 2007, chegando a atingir 316.301 toneladas e caindo um pouco para 312.348 toneladas em 2008 (IBGE, 2010). No território nacional, a plantação de goiabas concentra-se, principalmente, nas regiões Nordeste e Sudeste, as quais se configuraram como as mais produtoras, em 2008, com 135.016 e 125.201 toneladas, respectivamente (PEREIRA; RYOSUKE, 2011). A produção brasileira de goiaba destina-se quase que totalmente ao mercado interno, na produção de doces, sucos, geleia e polpa congelada, que, nos últimos oito anos, vem experimentando significativo aumento de oferta (IBGE, 2010).

A cultura da goiabeira vem sendo severamente ameaçada pela presença nos pomares do nematoide de galha da goiabeira, Meloidogyne enterolobii Yang e Eisenback (=M. mayaguensis), encontrado nas principais regiões produtoras, comprometendo a produção, chegando a causar a morte da planta (MARANHÃO et al., 2003; TORRES et al., 2005).

No Brasil, a espécie $M$. enterolobii foi assinalada pela primeira vez em Petrolina (PE), Curaçá e Maniçoba (BA), por Carneiro et al. (2001), causando danos severos em plantios comerciais de goiabeira. Nesse relato, essa espécie foi denominada de $M$. mayaguensis, entretanto atualmente tal espécie é considerada sinonímia de $M$. enterolobii, comprovada por estudos de dados morfológicos, gama de hospedeiros, fenótipos para as enzimas EST e MDH e sequências do mtDNA realizados por $\mathrm{Xu}$ et al. (2004).

A partir daí, esse nematoide vem sendo detectado parasitando goiabeiras em plantios comerciais nos Estados do Rio de Janeiro (LIMA et al., 2003), Rio Grande do Norte (TORRES et al., 2004), Ceará (TORRES et al., 2005), São Paulo (ALMEIDA et al., 2006), Paraná (CARNEIRO et al., 2006), Piauí (SILVA et al., 2006), Espírito Santo (LIMA et al., 2007), Mato Grosso do Sul (ASMUS et al., 2007), Santa Catarina e Rio Grande do Sul (GOMES et al., 2008) e Minas Gerais (NEVES; MONTEIRO, 2010).

Os danos e perdas causados por M. enterolobii em goiabeira são tão severos que, na região de Petrolina, ocorreu redução da área plantada de 6.000 ha para 2.500 ha (CARNEIRO et al., 2006). Uma vez instalado o nematoide na goiabeira, o controle torna-se difícil, sendo a melhor medida de controle o uso de porta-enxertos resistentes (CARNEIRO et al., 2007). Plantas atacadas pelo nematoide das galhas apresentam a formação de engrossamentos nas raízes, a paralisação do crescimento e a morte de pontas de raízes. As plantas podem apresentar depauperamento e declínio lento, deficiência nutricional, diminuição do tamanho das folhas e frutos, e redução da produtividade (GOMES et al., 2008).

As folhas são importantes centros metabólicos, e a análise foliar reflete o estado nutricional da planta com mais fidelidade. Por isso, a análise foliar é uma das melhores técnicas disponíveis para avaliar o estado nutricional das plantas (PRADO et al., 2008).

O objetivo deste trabalho foi identificar genótipos de goiabeira e de araçazeiro resistentes a M. enterolobii, com vistas à utilização como porta-enxertos compatíveis com as variedades comerciais de goiabeira e também obter o equilíbrio dos teores foliares de macronutrientes e micronutrientes das plantas avaliadas.

\section{MATERIAL E MÉTODOS}

O trabalho foi conduzido no Setor de Hidroponia do Departamento de Ciência do Solo e em casa de vegetação do Laboratório de Nematologia do Departamento de Fitopatologia da Universidade Federal de Lavras (UFLA), localizada no município de Lavras-MG, nas coordenadas " $21^{\circ} 13$ ' $55^{\prime}$ " S e “4457'43” W, à altitude de $925 \mathrm{~m}$. O clima é do tipo Cwb, segundo a classificação de Köppen (mesotérmico com verões brandos e suaves e estiagem de inverno).

As sementes foram coletadas de frutos fisiologicamente maduros de dois genótipos de goiabeira (P. guajava) e nove genótipos de araçazeiro (Psidium $\mathrm{sp}$.), oriundos de regiões próximas ao município de Lavras-MG. As sementes foram semeadas de duas a quatro em tubetes plásticos, com $5 \mathrm{~cm}$ de diâmetro e $20 \mathrm{~cm}$ de altura, contendo vermiculita como substrato. Em seguida, foram colocados em suportes próprios e transferidos para caixas rasas niveladas, aqui denominadas de piscinas, onde passaram a receber solução nutritiva proposta por Faquin e Chalfun (2009). A irrigação e a nutrição das plantas foram realizadas por capilaridade da própria vermiculita. Em todo o período experimental, foi utilizada solução nutritiva. A reposição de nutrientes na solução nutritiva do reservatório foi efetuada por meio da condutividade elétrica, ajustando-a diariamente pela adição de soluções-estoque de macro e micronutrientes, e o $\mathrm{pH}$ da solução nutritiva foi mantido entre 5,5 e 6,5.

Após as mudas alcançarem 8 a $10 \mathrm{~cm}$ de altura, foi realizado o desbaste deixando-se apenas as mais vigorosas. Ao atingirem, em média, $12 \mathrm{~cm}$ de altura, foram transferidas para casa de vegetação, onde foram transplantadas para vasos plásticos de 3,5 L, contendo Plantmax ${ }^{\circledR}$ como substrato. As temperaturas no interior da casa de vegetação, protegida com polietileno de $120 \mu \mathrm{m}$ de espessura, variaram-se de 18 a $38^{\circ} \mathrm{C}$. Quinze dias após a transferência, as mudas tiveram suas alturas aferidas e foram inoculadas com M. enterolobii, conforme procedimento a seguir:

Preparo do inóculo - O inóculo, cedido pela 
Embrapa Semiárido - CPATSA -Petrolina-PE, foi inoculado em raízes de tomateiro (Solanum lycopersicon Mill), linhagem 684, reconhecida como resistente a $M$. incognita (Kofoid \& White) Chitwood e M. javanica (Treub) Chitwood. Dois meses após a inoculação, as raízes dos tomateiros foram cuidadosamente retiradas do substrato, lavadas e cortadas em pequenos segmentos de 1-2 cm, seguindo-se a extração de ovos conforme a técnica descrita por Hussey e Barker (1973). O método consiste, basicamente, em acondicionar pedaços de raízes em recipiente de vidro com capacidade para $500 \mathrm{~mL}$, adicionando-se $200 \mathrm{~mL}$ de uma solução de hipoclorito de sódio a $0,5 \%$ e agitando-se no liquidificador por 40 segundos. A suspensão foi imediatamente passada em peneiras de 200 e 500 mesh, tipo "US Standard Series". Os ovos (e J2 estádio infectivo) que ficaram retidos na última peneira foram lavados em água corrente para a remoção dos resíduos de hipoclorito de sódio e transferidos, com a ajuda de uma pisseta, para um vidro com tampa plástica de $50 \mathrm{~mL}$. Dessa suspensão, foi retirado amostra de $1 \mathrm{~mL}$ para contagem dos ovos e J2 com auxílio da câmara de contagem de Peters, por meio do microscópio fotônico. A concentração da suspensão foi ajustada para $1.000 \mathrm{ovos} / \mathrm{mL}$.

Inoculação com o nematoide $M$. enterolobii - Separadas em três blocos, uniformizados pelo desenvolvimento fisiológico, as mudas dos araçazeiros e das goiabeiras foram inoculadas com 10.000 ovos/ planta após 15 dias do transplantio. O inóculo foi depositado em quatro pequenas depressões de $5 \mathrm{~cm}$ no solo, distanciados $2 \mathrm{~cm}$ do colo da planta, com auxílio de uma pipeta graduada e automática (Macroset). Decorridos 30 dias da primeira inoculação, foi realizada nova inoculação com 10.000 ovos/planta, seguindo a mesma metodologia, perfazendo-se então 20.000 ovos como população inicial (Pi). As plantas foram convenientemente espaçadas na casa de vegetação, recebendo regas quinzenais, de solução nutritiva proposta por Faquin e Chalfun (2008), e demais tratos culturais durante um período experimental de quatro meses.

Avaliação dos dados - A análise do comportamento dos hospedeiros, em relação ao parasitismo de M. enterolobii, ocorreu aos 120 dias após a inoculação. Para isso, as raízes das plantas foram processadas pela técnica de Hussey e Barker (1973), e a população de ovos, juvenis e adultos foi estimada com auxílio da câmara de contagem de Peters, por meio do microscópio fotônico. O delineamento utilizado foi o inteiramente casualizado, com 11 genótipos, uma espécie de nematoide e seis repetições, sendo duas plantas de cada genótipo adicionadas como controle, não sendo inoculadas. Os dados obtidos constituíram a população final (Pf) e foram utilizados na determinação do fator de reprodução (FR), conforme Oostenbrink (1966), definido pela relação Pf/Pi. Plantas com $\mathrm{FR}<1$ foram consideradas resistentes, e aquelas com FR $>1$, suscetíveis. A análise estatística da variável fator de reprodução foi realizada utilizando o software R (2008). A homogeneidade e a normalidade da distribuição amostral foram analisadas pelos testes de Bartlett e Shapiro-Wilks, respectivamente. Quando verificado que os dados não seguiam uma distribuição normal e não possuíam a mesma variância, realizou-se a análise de variância nãoparamétrica, usando o teste de Kruskal-Wallis, a 5\% de probabilidade.

Para a obtenção da matéria seca, as folhas foram colhidas e secas em estufa de circulação forçada de ar, a $60^{\circ}-65^{\circ} \mathrm{C}$, até peso constante e, posteriormente, pesadas. A matéria seca foi moída em moinho tipo Willey, com malha de 20 mesh. Após a moagem, os teores foliares dos macro e micronutrientes foram analisados quimicamente, de acordo com Malavolta et al. (1997). A variável teor foliar foi analisada estatisticamente pelo programa Sisvar (FERREIRA, 2000), realizando-se a análise de variância e o teste de Scott-Knott, a 5\% de probabilidade, para comparação de médias.

\section{RESULTADOS E DISCUSSÃO}

A produção média de ovos e $\mathrm{J} 2$ de $M$. enterolobii variou de 181,67 a 428.146,1, e o fator de reprodução (FR) médio variou de 0,02(ALU1) a 42,81(A-PASTO) (Tabela 1). Os genótipos de araçazeiro (Psidium sp.) AUFLA1, AUFLA4, AUFLA5 e APASTO e os genótipos de goiabeira (P. guajava) G-ROXA e G-AMA foram considerados suscetíveis. Os genótipos de araçá ALU1, ALU2, ALU3, AROXO-C e AROXO-U mostraram-se resistentes (Tabela 1).

Em pesquisas desenvolvidas por Almeida et al. (2009), fontes de resistência $a$ M. enterolobii foram encontradas entre os acessos pertencentes à Coleção de Fruteiras Nativas e Exóticas da UNESP/ FCAV, sendo os acessos 1;2 e 5 de Araçazeiro-Amarelo, pertencentes a espécie $P$. cattleyanum Sabine, os que mostraram alto grau de resistência genética quando inoculados com o referido nematoide. Os mesmos autores também verificaram que os acessos citados não foram compatíveis para enxertia com goiabeira 'Paluma', o que inviabiliza a utilização destes materiais como porta-enxerto para essa cultivar. Carneiro et al. (2007) identificaram três acessos de $P$. cattleyanum, provenientes de Pelotas - RS, resistentes a M. enterolobii. Ao que tudo indica, a fonte de resistência genética a $M$. enterolobii encontra-se, inicialmente, nos araçazeiros da espécie $P$. cattleyanum, e por serem muito recentes os estudos, é necessário avaliar outras espécies de araçazeiro.

Burla et al. (2007), Carneiro et al. (2007) e Scherer (2009) obtiveram elevados valores de fator de reprodução ao avaliarem materiais de goiabeira inoculados com esse nematoide. Até a presente data, 
resistência a $M$. enterolobii em genótipos de $P$. guajava ainda não foi relatada, fato este que prejudica a elaboração de programas de melhoramento.

Considerando o Brasil um dos centros de origem da goiabeira, mais screenings devem ser realizados com acessos de Psidium que obtiveram FR $<1$ para selecionar porta-enxertos com compatibilidade e sobrevivência em nível de campo, quando enxertados com cultivares comerciais, como 'Paluma' e 'Pedro Sato'.

Quantos aos teores foliares de macro e micronutrientes das plantas avaliadas (Tabela 2), não houve diferença significativa entre os genótipos quanto ao teor de $\mathrm{K}$.

Os genótipos A-UFLA1, A-PASTO, G-ROXA e G-AMAR, que foram bons hospedeiros (FR $>1$ ) de M. enterolobii (Tabela1), apresentaram menor teor foliar de N, P, Ca, Mg e Fe (Tabela 2), comparados com as plantas-controle. Entretanto, em relação ao nutriente $\mathrm{Mn}$, nos genótipos G-ROXO e G-AMAR, houve maior acúmulo comparado com os genótipos-controle (Tabela 2). Segundo Rossi e Ferraz (2005), com a alta infecção do sistema radicular das plantas, o juvenil toma a forma salsichoide, perde a mobilidade e passa a se alimentar da raiz, ocorrendo o parasitismo, onde resultam em severo comprometimento à absorção e ao transporte de água e nutrientes nas plantas. No entanto, os resultados obtidos no presente estudo estão de acordo com os referidos autores, onde apresentou menor absorção de nutrientes. Nos genótipos ALU1, ALU2, ALU3, AROXO-C e AROXO-U (Tabela 1) verificou-se FR $<1$, mostrando que esses genótipos são resistentes e que também apresentaram teores de macro e micronutrientes equilibrados, comparando-se com os genótipos-controle (SI) (Tabela 2).

Os genótipos AUFLA4 e AUFLA5, com FR $>1$ (Tabela 1), apresentaram menor teor foliar de $\mathrm{Mg}$, enquanto outros nutrientes mantiveram os teores equilibrados quando comparados aos genótipos-controle (SI) (Tabela 2).

A presença de fitonematoides no sistema radicular, além de diminuir a produção da planta, proporciona o declínio generalizado, com sintomas nas raízes (galhas e apodrecimento) e na parte aérea (bronzeamento, amarelecimento, queima dos bordos e queda das folhas). Por outro lado, o desequilíbrio de nutrientes pode predispor as plantas ao ataque de patógenos e contribuir para maior severidade de algumas doenças. Plantas bem nutridas suportam melhor a presença de patógenos. Os nutrientes afetam tanto o hospedeiro quanto o patógeno, de forma direta ou indiretamente (NATALE et al., 2009).

Com base nos dados apresentados na Tabela 3 , estabeleceu-se a ordem decrescente de teor para macro e micronutrientes, que poderão ser utilizados como referenciais de exigência nutricional de plantas de goiabeira (P. guajava) e araçazeiro (Psidium sp.) sem e com sintomas causados por $M$. enterolobii. Com base na referida tabela, observa-se que houve desbalanço nutricional na ordem de exigência de macronutrientes nos teores foliares dos genótipos AUFLA1, AUFLA4, AUFLA5, Goiabeira-roxa e Goiabeira- amarela quando comparados com os genótipos sem inoculação de $M$. enterolobii.

De acordo com Gomes et al. (2008), em goiabeiras, os sintomas causados por $M$. enterolobii estão associados à deficiência de nitrogênio, fósforo e potássio. Além disso, as plantas apresentam uma tendência a menos pela absorção de magnésio e acúmulo de manganês. No presente trabalho, os sintomas causados nas plantas infectadas com $M$. enterolobii estão em concordância com os autores, exceto para o K, que apresentou comportamento equilibrado.

Quanto aos micronutrientes, os genótipos AROXO-C, AROXO-U, Goiabeira-roxa, Goiabeira-amarela, mostraram uma tendência de acúmulo do nutriente $\mathrm{Mn}$, comparados com a ordem dos genótipos não inoculados com M. enterolobii (Tabela 3).

A composição mineral de plantas parasitadas por nematoides, usualmente, difere da composição de plantas não parasitadas. Essas alterações na composição mineral não seguem um padrão rígido, podendo ocorrer, em alguns casos, uma diminuição ou acúmulo ou, ainda, permanecerem inalterados os teores de determinados nutrientes (GOMES et al., 2008).

Dos 11 genótipos avaliados, seis de araçazeiro e dois de goiabeira, as fontes de resistência foram encontradas entre os araçazeiros, corroborando os resultados obtidos até então por outras pesquisas, buscando resistência a $M$. enterolobii em genótipos do gênero Psidium. Quanto à absorção de macro e micronutrientes pelos genótipos avaliados, observaram-se alterações no teor de alguns nutrientes pela presença do patógeno, mas as diferenças mais significantes estão entre os diferentes genótipos e não só pela presença ou ausência do nematoide. 
TABELA 1 - Número total de ovos (J2), fator de reprodução (FR) e reação (R=resistente; $\mathrm{S}=$ suscetível) em raízes de genótipos de araçazeiro e goiabeira inoculados com Meloidogyne enterolobii.

\begin{tabular}{cccccc}
\hline Genótipos & Nome comum & \multicolumn{2}{c}{ Nome científico N$^{\circ}$.Total de ovos (J2) } & FR & Reação \\
\hline AUFLA1 & Araçazeiro & Psidium sp. & $90.620,4$ & $9,02(45,50)^{1} \mathrm{~d}$ & $\mathrm{~S}$ \\
AUFLA4 & Araçazeiro & Psidium sp. & $28.271,8$ & $2,83(39,17) \mathrm{e}$ & $\mathrm{S}$ \\
AUFLA5 & Araçazeiro & Psidium sp. & $17.289,3$ & $1,73(33,83) \mathrm{f}$ & $\mathrm{S}$ \\
ALU1 & Araçazeiro & Psidium sp. & 200 & $0,02(8,67) \mathrm{h}$ & $\mathrm{R}$ \\
ALU2 & Araçazeiro & Psidium sp. & 1450 & $0,15(22,25) \mathrm{g}$ & $\mathrm{R}$ \\
ALU3 & Araçazeiro & Psidium sp. & 2755 & $0,28(26,5) \mathrm{g}$ & $\mathrm{R}$ \\
A-PASTO & Araçazeiro & Psidium sp. & $428.146,1$ & $42,81(62,4) \mathrm{a}$ & $\mathrm{S}$ \\
AROXO-C & Araçazeiro & Psidium sp. & 200 & $0,02(8,00) \mathrm{h}$ & $\mathrm{R}$ \\
AROXO-U & Araçazeiro & Psidium sp. & 181,67 & $0,18(12,08) \mathrm{h}$ & $\mathrm{R}$ \\
G-ROXA & Goiabeira-roxa & Psidium guajava & $299.366,3$ & $29,94(57,17) \mathrm{b}$ & $\mathrm{S}$ \\
G-AMAR & Goiabeira-amarela & Psidium guajava & $222.079,3$ & $22,21(52,33) \mathrm{c}$ & $\mathrm{S}$ \\
\hline
\end{tabular}

As médias seguidas pela mesma letra não diferem entre si, pelo teste de Kruskal Wallis, ao nível de $5 \%$ de probabilidade. ${ }^{1}$ Rank: Classificação obtida conforme metodologia de Kruskal-Wallis;

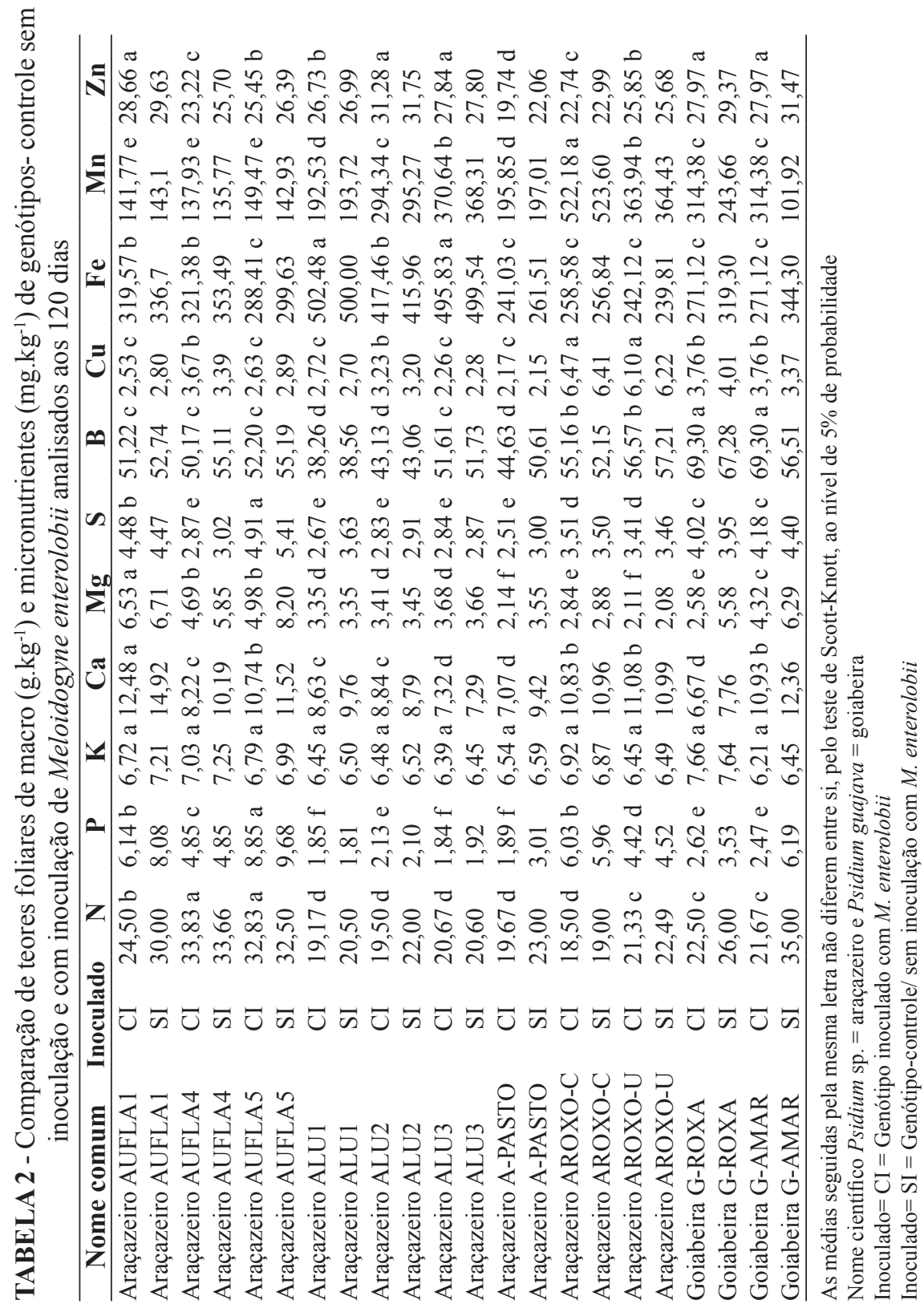


TABELA 3 - Teores foliares de macronutrientes $\left(\mathrm{g} \cdot \mathrm{kg}^{-1}\right)$ e micronutrientes $\left(\mathrm{mg} \cdot \mathrm{kg}^{-1}\right)$ em ordem decrescente de genótipos-controle sem inoculação(SI) e com inoculação (CI) de Meloidogyne enterolobii, analisados aos 120 dias.

\begin{tabular}{lcll}
\hline \multicolumn{1}{c}{ Nome comum } & Inoculado & $\mathrm{Macronutrientes}$ & Micronutrientes \\
Araçazeiro AUFLA1 & SI & $\mathrm{N}>\mathrm{Ca}>\mathrm{K}>\mathrm{P}>\mathrm{Mg}>\mathrm{S}$ & $\mathrm{Fe}>\mathrm{Mn}>\mathrm{B}>\mathrm{Zn}>\mathrm{Cu}$ \\
Araçazeiro AUFLA1 & CI & $\mathrm{N}>\mathrm{Ca}>\mathrm{K}>\mathrm{Mg}>\mathrm{P}>\mathrm{S}$ & $\mathrm{Fe}>\mathrm{Mn}>\mathrm{B}>\mathrm{Zn}>\mathrm{Cu}$ \\
Araçazeiro AUFLA4 & SI & $\mathrm{N}>\mathrm{Ca}>\mathrm{K}>\mathrm{Mg}>\mathrm{P}>\mathrm{S}$ & $\mathrm{Fe}>\mathrm{Mn}>\mathrm{B}>\mathrm{Zn}>\mathrm{Cu}$ \\
Araçazeiro AUFLA4 & CI & $\mathrm{N}>\mathrm{Ca}>\mathrm{K}>\mathrm{P}>\mathrm{Mg}>\mathrm{S}$ & $\mathrm{Fe}>\mathrm{Mn}>\mathrm{B}>\mathrm{Zn}>\mathrm{Cu}$ \\
Araçazeiro AUFLA5 & SI & $\mathrm{N}>\mathrm{Ca}>\mathrm{P}>\mathrm{Mg}>\mathrm{K}>\mathrm{S}$ & $\mathrm{Fe}>\mathrm{Mn}>\mathrm{B}>\mathrm{Zn}>\mathrm{Cu}$ \\
Araçazeiro AUFLA5 & CI & $\mathrm{N}>\mathrm{Ca}>\mathrm{P}>\mathrm{K}>\mathrm{S}>\mathrm{Mg}$ & $\mathrm{Fe}>\mathrm{Mn}>\mathrm{B}>\mathrm{Zn}>\mathrm{Cu}$ \\
Araçazeiro ALU1 & SI & $\mathrm{N}>\mathrm{Ca}>\mathrm{K}>\mathrm{Mg}>\mathrm{S}>\mathrm{P}$ & $\mathrm{Fe}>\mathrm{Mn}>\mathrm{B}>\mathrm{Zn}>\mathrm{Cu}$ \\
Araçazeiro ALU1 & CI & $\mathrm{N}>\mathrm{Ca}>\mathrm{K}>\mathrm{Mg}>\mathrm{S}>\mathrm{P}$ & $\mathrm{Fe}>\mathrm{Mn}>\mathrm{B}>\mathrm{Zn}>\mathrm{Cu}$ \\
Araçazeiro ALU2 & SI & $\mathrm{N}>\mathrm{Ca}>\mathrm{K}>\mathrm{Mg}>\mathrm{S}>\mathrm{P}$ & $\mathrm{Fe}>\mathrm{Mn}>\mathrm{B}>\mathrm{Zn}>\mathrm{Cu}$ \\
Araçazeiro ALU2 & CI & $\mathrm{N}>\mathrm{Ca}>\mathrm{K}>\mathrm{Mg}>\mathrm{S}>\mathrm{P}$ & $\mathrm{Fe}>\mathrm{Mn}>\mathrm{B}>\mathrm{Zn}>\mathrm{Cu}$ \\
Araçazeiro ALU3 & SI & $\mathrm{N}>\mathrm{Ca}>\mathrm{K}>\mathrm{Mg}>\mathrm{S}>\mathrm{P}$ & $\mathrm{Fe}>\mathrm{Mn}>\mathrm{B}>\mathrm{Zn}>\mathrm{Cu}$ \\
Araçazeiro ALU3 & CI & $\mathrm{N}>\mathrm{Ca}>\mathrm{K}>\mathrm{Mg}>\mathrm{S}>\mathrm{P}$ & $\mathrm{Fe}>\mathrm{Mn}>\mathrm{B}>\mathrm{Zn}>\mathrm{Cu}$ \\
Araçazeiro A-PASTO & SI & $\mathrm{N}>\mathrm{Ca}>\mathrm{K}>\mathrm{S}>\mathrm{Mg}>\mathrm{P}$ & $\mathrm{Fe}>\mathrm{Mn}>\mathrm{B}>\mathrm{Zn}>\mathrm{Cu}$ \\
Araçazeiro A-PASTO & $\mathrm{NI}>\mathrm{Ca}>\mathrm{K}>\mathrm{S}>\mathrm{Mg}>\mathrm{P}$ & $\mathrm{Fe}>\mathrm{Mn}>\mathrm{B}>\mathrm{Zn}>\mathrm{Cu}$ \\
Araçazeiro AROXO-C & SI & $\mathrm{N}>\mathrm{Ca}>\mathrm{K}>\mathrm{P}>\mathrm{S}>\mathrm{Mg}$ & $\mathrm{Fe}>\mathrm{Mn}>\mathrm{B}>\mathrm{Zn}>\mathrm{Cu}$ \\
Araçazeiro AROXO-C & $\mathrm{NI}>\mathrm{Ca}>\mathrm{K}>\mathrm{P}>\mathrm{S}>\mathrm{Mg}$ & $\mathrm{Mn}>\mathrm{Fe}>\mathrm{B}>\mathrm{Zn}>\mathrm{Cu}$ \\
Araçazeiro AROXO-U & SI & $\mathrm{N}>\mathrm{Ca}>\mathrm{K}>\mathrm{P}>\mathrm{S}>\mathrm{Mg}$ & $\mathrm{Fe}>\mathrm{Mn}>\mathrm{B}>\mathrm{Zn}>\mathrm{Cu}$ \\
Araçazeiro AROXO-U & $\mathrm{N}>\mathrm{Ca}>\mathrm{K}>\mathrm{P}>\mathrm{S}>\mathrm{Mg}$ & $\mathrm{Mn}>\mathrm{Fe}>\mathrm{B}>\mathrm{Zn}>\mathrm{Cu}$ \\
Goiabeira-roxa G-ROXA & $\mathrm{CI}$ & $\mathrm{Fe}>\mathrm{Mn}>\mathrm{B}>\mathrm{Zn}>\mathrm{Cu}$ \\
Goiabeira-roxa G-ROXA & SI & $\mathrm{N}>\mathrm{K}>\mathrm{S}>\mathrm{P}>\mathrm{Mg}$ & $\mathrm{Mn}>\mathrm{Fe}>\mathrm{B}>\mathrm{Zn}>\mathrm{Cu}$ \\
Goiabeira-amarela G-AMAR & $\mathrm{Mg}>\mathrm{P}$ & $\mathrm{Fe}>\mathrm{Mn}>\mathrm{B}>\mathrm{Zn}>\mathrm{Cu}$ \\
Goiabeira-amarela G-AMAR & SI & $\mathrm{N}>\mathrm{Ca}>\mathrm{K}>\mathrm{P}>\mathrm{S}>\mathrm{Mg}$ & $\mathrm{Mn}>\mathrm{Fe}>\mathrm{B}>\mathrm{Zn}>\mathrm{Cu}$ \\
\hline
\end{tabular}

\section{CONCLUSÕES}

1- Os genótipos de goiabeiras (Psidium guajava) avaliados são suscetíveis a Meloidogyne enterolobii, fato este que ameaça a continuidade dos pomares comerciais. Como a fonte de resistência a M. enterolobii é detectada em genótipos de araçazeiro, é fundamental testar novos materiais de $P$. guineense $\mathrm{Sw}$. e $P$. cattleyanum para serem usados como porta-enxertos para goiabeiras comerciais.

2- Os teores de macro e micronutrientes, de modo geral, sofrem redução quando os genótipos são inoculados com o fitonematoide, sendo as maiores reduções detectadas nos genótipos com valores mais elevados de FR.

\section{REFERÊNCIAS}

ALMEIDA, E. J.; SOARES, P. L. M.; SANTOS, J. M.; MARTINS, A. B. G. Ocorrência de Meloidogyne mayaguensis na cultura da goiaba (Psidium guajava) no Estado de São Paulo. Nematologia Brasileira, Campinas, v. 30, n. 2, p.112- 113, 2006.

ALMEIDA, E.J.; SOARES, P.L.M.; SANTOS, J.M.; MARTINS, A.B.G. Resistência de goiabeiras e araçazeiros a Meloidogyne mayaguensis. Pesquisa Agropecuária Brasileira, Brasília, v.44, n.4, p.421423, 2009.

ASMUS G.L.; VICENTINI, E.M.; CARNEIRO, R.M.D.G. Ocorrência de Meloidogyne mayaguensis em goiabeira no Estado de Mato Grosso do Sul. Nematologia Brasileira, Campinas, v.30, n.3, p.112, 2007. 
BURLA, R.S.; SOUZA, R.M.; GONCALVES JR, E.; MOREIRA, F.O.M. Reacao de acessos de Psidium spp. a Meloidogyne mayaguensis. In: CONGRESSO BRASILEIRO DE NEMATOLOGIA, 27., 2007, Goiânia. Anais... CD-ROM.

CARNEIRO, R.G.; MONACO, A.P.A.; MORITZ, M.P.; NAKAMURA, K.C.; SCHERER, A. Identificacao de Meloidogyne mayaguensis em goiabeira e em plantas invasoras, em solo argiloso, no Estado do Paraná. Nematologia Brasileira, Campinas,, v.30, n.3, p.293-298, 2006.

CARNEIRO, R.M.D.G.; CIROTTO, P.A.; QUINTANILHA, A.P.; SILVA, D.B.; CARNEIRO, R.G. Resistance to Meloidogyne mayaguensis in Psidium spp. accessions and their grafting compatility with P. guajava cv. Paluma. Fitopatologia Brasileira, Brasília, v.32, n.4, p.281-284, 2007.

CARNEIRO, R.M.D.G.; MOREIRA, W.A.; ALMEIDA, M.R.A.; GOMES, A.C.M.M. Primeiro registro de Meloidogyne mayaguensis em goiabeira no Brasil. Nematologia Brasileira, Campinas, v.25, n2, p.223-228, 2001.

FAQUIN, V.; CHALFUN, N.N.J. Hidromudas: processo de produção de porta-enxerto de mudas frutíferas, florestais e ornamentais enxertadas em hidroponia (BRN.PI 0802792-7). Rio de Janeiro: INPI, 2008. Disponível em: <http://www.patentesonline. com.br/hidromudas-processo-de-producao-de-porta-enxertos-e-mudas-frutiferas-florestais-e-226848i. html>. Acesso em: 12 ago. 2010.

FERREIRA, D.F. Análise estatística por meio do SISVAR para Windows 4.0. In: REUNIÃO ANUAL DA REGIÃO BRASILEIRA DA SOCIEDADE INTERNACIONAL DE BIOMETRIA, 45., 2000, São Carlos. Anais... São Carlos: UFSCAR, 2000. p.255-258.

GOMES, V. M.. Meloidoginose da goiabeira: estudos sobre a sua patogênese e formas de convívio com a doença no campo. 2007. 80f. Dissertação (Mestrado) - Universidade Estadual do Norte Fluminense, Campos dos Goytacazes, 2007.

GOMES, V.M.; SOUZA, R.M.; SILVA, M.M.; DOLINSKI, C. Caracterização do estado nutricional de goiabeiras em declínio parasitadas por Meloidogyne mayaguensis. Nematologia Brasileira, Campinas, v.32, n.2, p.154-160, 2008.
HUSSEY, R.S.; BARKER, K.R. A comparison of methods of collecting inoculated of Meloidogyne spp., including a new technique. Plant Disease Reporter, Beltsville, v.57, n.4, p.1025-1028, 1973.

IBGE - Instituto Brasileiro de Geografia e Estatística. Produção agrícola municipal. Rio de Janeiro. Disponível em: <www.sidra.ibge.gov.br $>$. Acesso em: 20 set. 2010.

LIMA, I.M.; DOLINSKI, C.M.; SOUZA, R.M. Dispersão de Meloidogyne mayaguensis em goiabais de São João da Barra (RJ) e relato de novos hospedeiros dentre plantas invasoras e cultivadas. In: CONGRESSO BRASILEIRO DE NEMATOLOGIA, 27., 2003, Petrolina. Anais... p.257-258.

LIMA I.M.; MARTINS.; M.V.V.; SERRANO, L.A.L.; CARNEIRO, R.M.D.G. Ocorrência de $M e$ loidogyne mayaguensis em goiabeira cv 'Paluma' no estado do Espírito Santo. Nematologia Brasileira, Campinas, v.31, p. 133, 2007.

MALAVOLTA, E.; VITTI, G. C.; OLIVEIRA, S. A. Avaliação do estado nutricional das plantas: princípios e aplicações. 2.ed. Piracicaba: Potafos, 1997. 319 p.

MARANHÃO, S. R. V. L.; MOURA, R. M.; PEDROZA, E. M. R. Reação de indivíduos segregantes de araçazeiro a Meloidogyne incognita, $M$. javanica e M. mayaguensis. Nematologia Brasileira, Campinas, v. 27, n. 2, p. 173-178, 2003.

NATALE, W.; ROZANE, D. E.; SOUZA, H. A.; AMORIM, D. A. Cultura da goiaba: do plantio à comercialização. Jaboticabal: UNESP, 2009. 284p.

NEVES, W. S.; MONTEIRO, T. S. A. Primeiro Relato de Meloidogyne enterolobii em Goiabeira na Região de Jaíba, Norte de Minas Gerais. Revista Trópica - Ciências Agrárias e Biológicas, Chapadinha, v. 4, n. 2, p. 11-11, 2010.

OOSTENBRINK, M. Major characteristic of the relation between nematodes and plants. Wageningen: Medelingen Landbowhogeschool, 1966. p.46.

PRADO, R. de M.; ROZANE, D. E.; VALE, D. W. do; CORREIA, M. A. R.; SOUZA, H. A. de. Nutrição de plantas: diagnose foliar em grandes culturas. Jaboticabal: FCAV, Capes/Fundunesp, 2008. 301 p. 
PEREIRA, F. M.; RYOSUKE, K. Contribuição da pesquisa cinetífica brasileira no desenvolvimento de algumas frutíferas de clima subtropical. Revista Brasileira de Fruticultura, Jaboticabal. v. 33, n.1 - edição especial. p. 92 -108, 2011.

R. DEVELOPMENT CORE TEAM. R: A language and environment for statistical computing, reference index version 2.8.0. R Foundation for Statistical Computing. Vienna, 2008.

ROSSI, C. E.; FERRAZ, L. C. C. B. Fitonematoides da superfamilia Criconematoidea e Dorylaimoidea associados a fruteiras de clima subtropical e temperado nos estados de São Paulo e Minas Gerais. Nematologia Brasileira, Campinas, v.29, n.2, p.183-192, 2005.

SCHERER, A. Ocorrência e hospedabilidade de Meloidogyne mayaguensis em goiabeiras e em plantas de cobertura de solo no Paraná. 2009. 64 f. Tese (Doutorado) - Universidade Estadual de Londrina, Londrina, 2009.
SILVA, G.S.; ATAYDE SOBRINHO, C.; PEREIRA, A.L.; SANTOS, J.M. Ocorrência de Meloidogyne mayaguensis em goiabeira no Estado do Piauí. Nematologia Brasileira, Campinas, v.30, n.2, p.307309, 2006.

TORRES, G.R.C.; COVELLO, V.N.; SALES JUNIOR, R.; PEDROSA, E.M.R.; MOURA, R. Meloidogyne mayaguensis em Psidium guajava no Rio Grande do Norte. Fitopatologia Brasileira, Brasília, v.29, n.5, p.570, 2004.

TORRES, G.R.C.; SALES- JUNIOR, R.; NERIVANIA, V.; REHN, C.; PEDROSA, E.M.R.; R.M. MOURA. Ocorrência de Meloidogyne mayaguensis em goiabeira no Estado do Ceara. Nematologia Brasileira, Campinas, v.29, n.1, p.105-107, 2005.

XU, J.; PEILEI, L.; QINGPENG, M.; HAI, L. Characterization of Meloidogyne species from China using isozyme phenotypes and amplified mitochondrial DNA restriction fragment length polymorphism. European Journal of Plant Pathology, Dordrecht, v.110, p.309-315, 2004. 